\title{
PERFIL DE UTILIZAÇÃO DE ANTICONCEPCIONAL DE EMERGÊNCIA EM SERVIÇOS DE ATENDIMENTO FARMACÊUTICO DE UMA REDE DE FARMÁCIAS COMUNITÁRIAS
}

\author{
Márcio de Souza Cavalcante, Marília Almeida Soares, \\ Cristiane Macêdo Feijó, Marta Maria de França Fonteles* \\ Universidade Federal do Ceará \\ *E-mail: martafonteles@yahoo.com.br
}

\author{
Submetido em: 24/09/2015 \\ Aceito em: 10/05/2016 \\ Publicado em: 30/09/2016
}

\section{Resumo}

A contracepção de emergência é um método utilizado para prevenir a gravidez após a relação sexual desprotegida. O fácil acesso a esse método, sem nenhuma orientação, levanta preocupações em relação ao seu uso, principalmente no que diz respeito a uma possível substituição de um método contraceptivo regular por este, bem como pelo seu uso, em sua maioria, pelos adolescentes, e por generalização do uso repetitivo. Este estudo propôs avaliar o nível de conhecimento das mulheres usuárias, ou que tinham intenção de uso, do contraceptivo de emergência conhecido como "pílula do dia seguinte", permitindo um melhor acompanhamento da sua utilização, e disponibilização de dados farmacoepidemiológicos úteis para promoção do uso racional desse medicamento. Mulheres usuárias ou que tinham intenção de uso da pílula foram convidadas a participar da pesquisa sobre uso de contraceptivo de emergência. Foi aplicado um questionário e os dados foram incluídos em um programa estatístico específico. No total, foram realizadas 74 entrevistas, sendo 54 em um Serviço de Atendimento Farmacêutico, por telefone, ao cliente/consumidor, de uma rede de farmácias comunitárias, e 20 entrevistas aplicadas para clientes, no momento da dispensação do contraceptivo, em uma das filiais dessa Rede. A maioria das entrevistadas pretendia fazer uso ou utilizar contraceptivo de emergência sem orientação/prescrição médica. Elas relataram que obtiveram informações sobre esse medicamento por meio de amigos, familiares e da mídia (televisão, internet). A maioria informou ter utilizado o contraceptivo de emergência uma ou duas vezes. Um dos principais motivos para justificar a utilização da pílula do dia seguinte foi o sexo desprotegido. Nossos achados alertam para que se tenha uma maior preocupação em relação à possibilidade de uso indevido desse medicamento, e seu fácil acesso nas farmácias comunitárias.

Palavras-chave: Contraceptivo de emergência, Farmácia comunitária, Serviço de Atendimento farmacêutico

\section{Profile of emergency contraceptive use in pharmaceutical care} services operated by a community pharmacy network

\section{Abstract}

Emergency contraception is a method used to prevent pregnancy after unprotected intercourse. Easy access to this method without any guidance raises concerns regarding its possible repeated use, mostly by teenagers, instead of a regular contraceptive method. The aim of this study was to evaluate the level of knowledge of female users of the 'morning-after pill' to allow better monitoring of its use and to provide useful pharmacoepidemiological data to promote rational use of emergency contraception. Women who already used or intended to take the pill were invited to participate in the survey on emergency contraceptive use. We applied a questionnaire and the data were included in a statistical specific program. In total, 74 interviews were conducted, 54 by phone by a Pharmaceutical Care Service of the community pharmacies network, and 20 interviews in person at one of these pharmacies during the dispensing of the medicine. The majority of respondents informed us that some either intended to use or used emergency contraception without guidance or a prescription. They reported that they obtained information about 
the morning-after pill from friends, family and the internet. Most respondents reported having used emergency contraception once or twice. One of the main reasons to justify the use of the morning-after pill was unprotected sex. These observations signal possible concerns about improper use of this medicine which is easily accessible in community pharmacies.

Keywords: Emergency contraception, Community pharmacy, Pharmaceutical Care Service

\section{Perfil del uso de anticonceptivos de emergencia en servicios de atención farmacéutica de una red de farmacias comunitarias}

\section{Resumen}

La anticoncepción de emergencia es un método utilizado para prevenir el embarazo después del coito sin protección. El fácil acceso a este método, sin ninguna orientación, plantea preocupaciones con respecto a su uso, especialmente, sobre la posible sustitución de un método anticonceptivo regular por este, así como por su uso, sobre todo por los adolescentes, y por la generalización de su uso repetitivo. Este estudio propone analizar el nivel de conocimiento de las mujeres que usan, o tienen la intención de usar, el anticonceptivo de emergencia conocido como la "píldora del día después", lo que permite un mejor acompañamiento de su uso y disponibilidad de los datos de farmacoepidemiologicos útiles para promover el uso racional de ese medicamento. Mujeres usuarias o que tenían la intención de usar la píldora fueron invitadas a participar de la encuesta sobre el uso de anticonceptivos de emergencia. Se aplicó un cuestionario y los datos se incluyeron en un programa estadístico específico. En total, se realizaron 74 entrevistas, 54 en un Servicio de Atendimiento Farmacéutico telefónico, al cliente/consumidor, de una red de oficinas de farmacias comunitarias y 20 entrevistas aplicadas a clientes en el momento de la dispensación del anticonceptivo en una de las filiales de esa Red. La mayoría de las encuestadas tenían intención de usar el anticonceptivo de emergencia sin orientación/ prescripción médica. Ellas relataron que la informaron sobre ese medicamente fue obtenida a través de amigos, familiares y de los medios de comunicación (televisión, Internet). La mayoría informó que había usado la anticoncepción de emergencia una o dos veces. Una de las principales razones para justificar el uso de la píldora del día después era el sexo sin protección. Nuestros hallazgos advierten que se debe tener mayor preocupación a respecto de la posibilidad del mal uso de ese medicamento y de su fácil acceso en las farmacias comunitarias.

Palabras-clave: Anticoncepción de emergencia, Farmacia comunitaria, Servicio de Atención Farmacéutico

\section{INTRODUÇÃO}

A contracepção de emergência é um método utilizado para prevenir a gravidez após a relação sexual desprotegida. É também conhecida como "pílula do dia seguinte". Este método pode ser utilizado logo após a ocorrência de falha de outros contraceptivos e geralmente é eficaz se usado dentro de um curto espaço de tempo, após a exposição sexual(1). Esse método utiliza progestogênio na forma concentrada, e tem indicação reservada às situações especiais ou de exceção ${ }^{(1,2)}$. Entre as indicações, destacam-se: relação sexual sem uso de método anticonceptivo, falha conhecida ou presumida do método em uso de rotina, uso inadequado do anticonceptivo, e abuso sexual. Sempre que se fala em contracepção de emergência deve-se ressaltar seu caráter emergencial, não devendo, portanto, ser usada como método anticonceptivo regular, haja vista não ser eficaz para uso rotineiro(3). 
O fácil acesso à contracepção de emergência sem nenhuma orientação levanta preocupações em relação ao seu uso, principalmente no que diz respeito a uma possível substituição de um método contraceptivo regular por este método, ao seu uso, em sua maioria, pelos adolescentes, e à generalização do uso repetitivo(4)

A pílula de emergência, dependendo da fase do ciclo menstrual em que é usada, pode promover algumas mudanças no organismo da usuária, como: suprimir ou atrasar a ovulação; alterar a resposta endometrial na nidação e, por fim, pode alterar a fecundação devido à modificação do corpo lúteo e a motilidade tubária(4).

Apesar da pílula de emergência ter registros de eficácia como contraceptivo, uma vez iniciado o processo de nidação, o medicamento supracitado, como todos os fármacos contraceptivos, perde a efetividade. Desse modo, caso a nidação não possa ser impedida, existem riscos ao embrião ou à gravidez visto que foram documentadas poucas evidências de efeitos nocivos para o embrião, ou piora no prognóstico da gestação(4).

Os principais efeitos colaterais dos anticoncepcionais combinados são as náuseas e vômitos. Já os regimes com progestogênios puros apresentam uma incidência menor desses efeitos colaterais. Quando os vômitos ocorrem até duas horas após a ingestão, a dose deve ser repetida. Outros efeitos secundários, associados com ambos os regimes, em porcentagens menores, incluem: cansaço, aumento da sensibilidade das mamas e cefaleia, geralmente com duração menor do que 24 horas. A maioria das pacientes tratadas sangra 14 a 21 dias após o tratamento(5).

Apesar da disponibilidade de métodos contraceptivos, a incidência da gravidez indesejada ainda é muito elevada em todo o mundo, particularmente em países em desenvolvimento. De fato, na América Latina e no Caribe, tem sido referido que as mulheres têm mais filhos do que desejariam ter. Isso pode ser explicado por que milhões de pessoas têm necessidades não satisfeitas de planejamento familiar, seja por falta de acesso a métodos anticonceptivos apropriados ou por informação e apoio insuficientes para utilizá-los. No Brasil, esse problema alcança quase oito milhões de pessoas(6).

Sabe-se também que a contracepção de emergência não oferece qualquer proteção contra as infecções sexualmente transmissíveis (IST) ou contra o vírus da imunodeficiência humana (HIV). É fundamental que essa premissa seja conhecida pelos profissionais de saúde e, principalmente, pelos casais que utilizam a anticoncepção de emergência. Contudo, este não é problema exclusivo ou específico da anticoncepção de emergência. Outros métodos anticoncepcionais, com exceção dos preservativos masculino e feminino, enfrentam a mesma limitação e também não protegem contra as IST/HIV. Mesmo assim, continuam indicados, com critérios bem definidos, sem que se deixe de observar todos os cuidados necessários de aconselhamento para prevenção das IST/HIV. De certa forma, o mesmo ocorre com a anticoncepção de emergência. Porém, o fato desse método não proteger a mulher das IST/HIV, evidentemente, desperta a preocupação de profissionais de saúde. O que se teme, principalmente, é que ocorra o uso abusivo ou indiscriminado do método. Com isso, cogita-se que poderia ocorrer o abandono da camisinha ou a redução expressiva de seu uso, aumentando, perigosamente, a exposição para as IST/HIV $\left.{ }^{7}, 16\right)$.

Foi com o intuito de conhecer melhor as práticas contraceptivas adotadas por clientes/consumidoras, que requerem o Serviço de Atendimento Farmacêutico, de abrangência nacional, de uma grande rede de farmácias comunitárias, bem como observar aspectos relacionados à dispensação da pílula do dia seguinte em farmácia comunitária, que este estudo foi proposto. Igualmente, o estudo se propõe a apresentar o nível de conhecimento das mulheres usuárias ou que tinham intenção de uso do contraceptivo de emergência, permitindo um melhor acompanhamento de sua utilização, e fornecendo dados farmacoepidemiológicos que poderão ser úteis para promoção do uso racional do medicamento.

\section{METODOLOGIA}

Trata-se de um estudo do tipo observacional, descritivo e transversal, na área de Farmacoepidemiologia, particularmente envolvendo o Estudo de Utilização de Medicamentos. 
A pesquisa foi, inicialmente, realizada no Serviço de Atendimento Farmacêutico ao Consumidor (SAC FARMA/SAC), nos anos de 2008 e 2009. O SAC é um serviço vinculado a uma grande rede de farmácias comunitárias que presta orientação farmacêutica gratuita por telefone, para diferentes regiões do Brasil.

A atualização da pesquisa desenvolvida pelo SAC FARMA foi realizada em uma filial pertencente à mesma rede de farmácias comunitárias nos anos de 2013 a 2014. Os dois locais envolvidos no estudo estão situados no município de Fortaleza, Ceará.

Antes da aplicação dos questionários, nos dois locais da pesquisa, foi feito um treinamento com os auxiliares de farmácia, farmacêuticos e estagiários, de forma a otimizar o instrumento e padronizar os registros.

No total, foram entrevistadas 54 mulheres, que eram clientes atendidas pelo SAC FARMA, utilizando-se questionário estruturado para a pesquisa. Foram incluídas, no estudo, clientes do sexo feminino, que concordaram com a pesquisa, e demonstraram ter intenção de uso ou ser usuária do contraceptivo de emergência. O mesmo questionário foi aplicado a 20 mulheres clientes de uma das filiais da rede de farmácias mencionada, e obedeceu aos mesmos critérios de inclusão estabelecidos para aplicação do questionário no SAC. Utilizou-se uma amostragem de conveniência.

Os dados dos atendimentos realizados no SAC FARMA foram armazenados no Tactium Manegement ${ }^{\oplus}$, software com capacidade para gerar um banco de dados que organiza informações de forma eficiente ao desenvolvimento da pesquisa.

Após o atendimento e esclarecimento da informação solicitada sobre contraceptivo de emergência, as usuárias do SAC foram convidadas a participar de uma pesquisa sobre a utilização da "Pílula do Dia Seguinte", por meio da aplicação de um questionário, não sendo obrigatória a identificação da usuária do serviço. Com a preocupação de não influenciar nas respostas das participantes, foi utilizado um layout sucinto, com perguntas abertas, deixando-as responder livremente o questionário.

Foram considerados os registros de atendimentos farmacêuticos sobre "Pílula do Dia Seguinte" de outubro de 2008 a maio de 2009, no SAC FARMA. Os entrevistadores asseguraram às usuárias a possibilidade de não participar do estudo, a possibilidade de abandonar o preenchimento do questionário, a qualquer momento, ou não responder questões que considerassem inconvenientes de responder. Garantiram, também, o anonimato daquelas que concordassem em participar da pesquisa.

Quanto aos aspectos éticos, o estudo foi projetado de acordo com as diretrizes e normas regulamentadoras de pesquisa envolvendo seres humanos (CNS 466/12), e foi aprovado pelo Comitê de Ética em Pesquisa, da Universidade Federal do Ceará.

Os dados foram tabulados e analisados no Sigma Plot 11.0, e Sigma Stat 3.5. A análise descritiva ocorreu por meio do cálculo das médias das variáveis, com análises comparativas. Os testes Qui-Quadrado de Independência e o teste Exato de Fisher foram realizados para verificar se existia associação entre as variáveis em estudo, considerando-se significância estatística quando $p<0,05$.

\section{RESULTADOS E DISCUSSÃO}

O estudo avaliou o perfil socioeconômico de mulheres usuárias ou que tinham intenção de uso, bem como o nível de conhecimento em relação à contracepção de emergência, incluindo aspectos relacionados aos seus riscos e da prescrição.

Entre as entrevistadas pelo SAC FARMA, das 54 clientes estudadas, 35 relataram não ser a primeira vez que faziam a utilização da "Pílula do Dia Seguinte", enquanto que apenas sete, das 20 mulheres entrevistadas na farmácia filial da Rede, relataram já ter feito uso, perfazendo $64,8 \%$ e $35,0 \%$, respectivamente, valores estatisticamente diferentes (teste Exato de Fisher). 0 fato de menos usuárias da farmácia já terem utilizado o medicamento anteriormente, pode inferir que a Farmácia, como estabelecimento de promoção da saúde, ainda é requerida para o esclarecimento de dúvidas na primeira aquisição do medicamento.

As indicações da contracepção de emergência são reservadas a situações especiais e excepcionais, a fim de prevenir gravidez inoportuna ou indesejada após relação que, por alguma razão, foi desprotegida. Entre as principais indicações de contracepção de emergência está a relação sexual sem uso de método anticonceptivo, falha conhecida ou presumida do 
método em uso de rotina, uso inadequado do anticonceptivo e abuso sexual. A contracepção de emergência não deve ser usada de forma planejada, previamente programada, ou substituir método anticonceptivo como rotina(8).

Os principais motivos da intenção de uso entre as entrevistadas foram sexo desprotegido ( $51,8 \%$ no SAC, e 45,0\% na farmácia comunitária), seguido do esquecimento de tomada do anticoncepcional de rotina (21,4\% no SAC, e 20,0\% na farmácia comunitária) e rompimento do preservativo (19,6\% no SAC, e 15\% na farmácia comunitária). Outras razões indicadas foram o fato da entrevistada estar no intervalo do método anticoncepcional regular ou por resposta a exame hormonal (Figura 1). Esses dados corroboram estudo ${ }^{(12)}$ em que, entre as usuárias de contracepção de emergência, 61,6\% alegaram uso deste método devido ao não uso de preservativo, sendo que $12,5 \%$, inclusive, afirmaram que o utilizaram devido à realização mal sucedida do coito interrompido(8).

Muitas mulheres estão sujeitas a gestações não planejadas, sendo, adolescentes e jovens, as mais propícias a estas gestações ${ }^{(9)}$. Existe uma preocupação específica em relação a essa idade, pois gestações cada vez mais precoces podem causar altos índices de complicações obstétricas e psicológicas; além disso, é uma fase na qual pode ocorrer prática de uma sexualidade sem responsabilidade, baseada, muitas vezes, no prazer momentâneo( ${ }^{(8)}$. Neste estudo, as entrevistadas encontravam-se na faixa etária entre 16 e 50 anos, sendo que houve predominância de mulheres com idade entre 23 e 29 anos no SAC (44,4\%), e entre 30 e 36 anos, na Farmácia (45,0\%). Ressalta-se que, 29,6\% das entrevistadas (no SAC) e, 40,0\% das entrevistadas na Farmácia filial, tinham entre 16 e 22 anos (Figura 2).

Figura 1. Motivo da Utilização da Contracepção de Emergência

Figura 2. Faixa Etária das Entrevistadas
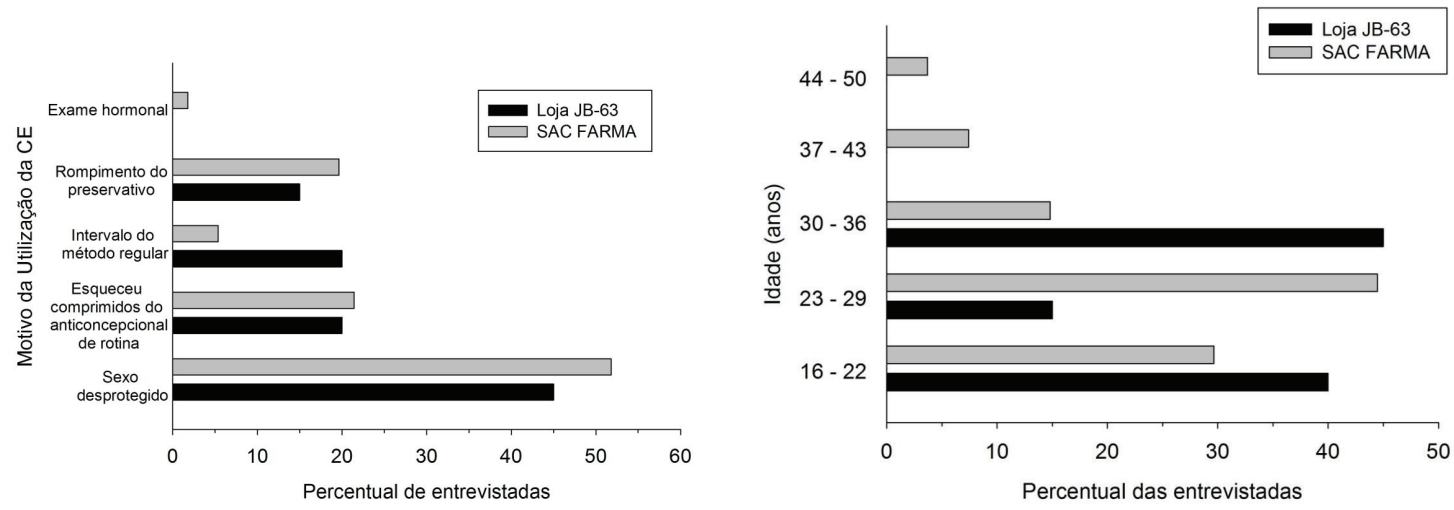

Para o Ministério da Saúde, a prescrição do método de contracepção de emergência, nas situações de exposição ao risco iminente de gravidez (violência sexual, falha do método em uso ou na sua utilização, não estar usando anticoncepcional), constitui-se dever do médico e um direito do adolescente. Contudo, no nosso país, mesmo com todas as diretrizes do Ministério da Saúde e do Conselho Federal de Medicina, a prescrição deste método pelos profissionais de saúde, tanto no setor público, quanto no setor privado, ainda é bastante restrita e mistificada(10). HARDY et al., 2001, também enfatizaram que todas as pessoas deveriam saber da existência da contracepção de emergência, mas não deveria ser possível usá-la sem uma avaliação profissional, preferencialmente realizada por médico ${ }^{(11)}$. Isto se torna claro ao avaliarmos nossos resultados, nos quais a maioria das mulheres, que utilizou ou pretendiam fazer uso da "Pílula do Dia Seguinte", entrevistadas no SAC (92,6\%) e na Farmácia (95,0\%), relatou não ter buscado orientação médica quando questionadas no momento da entrevista. 
A informação sobre a "Pílula do Dia Seguinte" foi obtida, principalmente, por outros meios do que por orientação/ prescrição médica. A maior parte das entrevistadas pelo SAC obteve informações sobre o método por meio de amigos ou familiares, enquanto que a maioria das entrevistadas na Farmácia obteve informações pela mídia (Tabela 1).

Tabela 1. Meio de Informação Sobre Contracepção de Emergência

\section{Locais de Conhecimento}

Mídia

\begin{tabular}{ll}
\hline Mídia & 16,0 \\
\hline Família/Amigos & 5 \\
\hline Internet & 4,0 \\
\hline Farmácia & 14,0 \\
\hline Outros & 12,0
\end{tabular}

Figueiredo et al. (2008) verificaram, em seu estudo, que um número predominante de entrevistados relatou já ter ouvido falar da contracepção de emergência, principalmente por meio de amigos e conhecidos (65,2\%), na escola (48,7\%), e na televisão $(47,4 \%)^{(9)}$. Os serviços e profissionais de saúde foram referidos como meio de informação sobre esse método por apenas $20,2 \%$ dos estudantes, e as farmácias, por $16,1 \%$.

Para Figueiredo (2004), a facilidade de aquisição do método em farmácias, para a compra, torna contínua a discussão sobre o acesso ao contraceptivo. Problemas culturais e informações distorcidas sobre contracepção de emergência constituem barreiras para sua aceitação, acesso e uso adequado(12).

Constatou-se que a maior parte das entrevistadas faz parte dos estratos socioeconômicos médio e baixo. A Figura 3 mostra que $77,8 \%$ das entrevistadas pelo SAC, e todas as entrevistadas na farmácia tem renda inferior a três salários mínimos, o que pode justificar certa dificuldade para aquisição do medicamento. Nesse caso, o contraceptivo poderia, então, ser adquirido em serviço público, pois, em 2005, o governo federal resolveu ampliar, em 57\%, a distribuição da contracepção de emergência nos serviços públicos, com a intenção de fornecer a chamada "Pílula do Dia Seguinte" a todas as mulheres, não somente às vítimas de violência sexual ${ }^{(13)}$. Figueiredo (2004) relata que os serviços de saúde pública no Brasil não acataram as normas de 1996 e de 1998, pois não foram iniciados os processos de divulgação, de fornecimento e de administração do método. Exceção a esta regra, foram os serviços de atendimento às vítimas de violência sexual, que mantiveram a implementação da normatização de 1998, e persistiram na luta pela difusão e disponibilização do método ${ }^{(12)}$.

Figura 3. Faixa Salarial das Entrevistadas

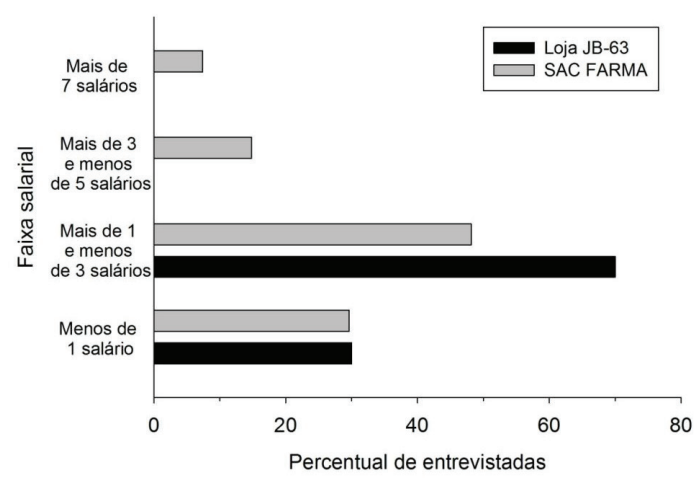


Quando questionadas sobre o conhecimento de métodos alternativos, a maioria das mulheres afirmou conhecer o preservativo e anticoncepcional oral, métodos comuns e bastante utilizados (Tabela 2).

Tabela 2. Conhecimento de outros métodos contraceptivos

\begin{tabular}{|lcccc|}
\hline \multirow{2}{*}{ Método } & Conhece (\%) & \multicolumn{2}{c|}{ Não conhece (\%) } \\
& SAC & Loja JB-63 & SAC & Loja JB-63 \\
\hline Preservativo & 92,6 & 100,0 & 7,4 & - \\
\hline Anticoncepcional Oral & 88,9 & 100,0 & 11,1 & - \\
\hline Anel Vaginal & 18,5 & 5,0 & 81,5 & 95,0 \\
\hline Adesivo Transdérmico & 16,7 & 5,0 & 83,3 & 95,0 \\
\hline Tabelinha & 37,0 & 5,0 & 63,0 & 95,0 \\
\hline Implante Subdérmico & 9,3 & - & 90,7 & 100,0 \\
\hline Anticoncepcional Injetável & 53,7 & - & 46,3 & 100,0 \\
\hline DIU & 40,7 & 20,0 & 59,3 & 80,0 \\
\hline Cirúrgico (L.Trompas/Nasectomia) & 22,2 & 25,0 & 77,8 & 75,0 \\
\hline Muco Cervical & 11,1 & - & 88,9 & 100,0 \\
\hline
\end{tabular}

Farmacêuticos exercem um papel fundamental para a promoção do uso racional de medicamentos. Sabendo-se que ele é o último profissional a ter contato com o paciente no ciclo da medicação, ele tem como dever auxiliar e impedir o uso indevido de medicamentos, evitando, assim, problemas relacionados aos medicamentos, a ocorrência de automedicação, e consequentes reações adversas causadas pelos medicamentos ${ }^{(14)}$

Incluir a contracepção de emergência em normas técnicas de atendimento sem dúvida é algo importante. No entanto, os setores públicos deveriam adotar outras medidas que incorporassem a pílula de contracepção de emergência dentro dos direitos sexuais e reprodutivos. Todas as mulheres em idade reprodutiva, e com risco de gravidez indesejada, deveriam ter garantia de acesso à informação e ao uso desse medicamento. Os serviços de saúde, desde a atenção básica, deveriam estar preparados para oferecer resposta a essa demanda. Capacitação de profissionais de saúde para prescrição e aconselhamento, facilitação do acesso aos serviços de saúde e programas de educação, e informação para as usuárias são algumas medidas necessárias(15,16).

\section{CONCLUSÃO}

A maior parte das usuárias do método de contracepção de emergência, representado pelo uso da "pílula do dia seguinte", concentrou-se na faixa etária relacionada às adolescentes e jovens, com baixo ou médio poder econômico, e pretendia fazer uso ou utilizar a contracepção de emergência sem orientação/prescrição médica. As informações sobre a pílula eram obtidas por meio de amigos, familiares e mídia. Tal fato é preocupante ao se pensar no possível uso indevido e fácil acesso ao medicamento nas farmácias comerciais.

Constatou-se, também, que sexo desprotegido, como motivo de utilização da pílula do dia seguinte, possuía distribuição homogênea em todas as faixas etárias.

Ainda, nossas descobertas mostraram que os preservativos e os anticoncepcionais orais são os métodos contraceptivos mais conhecidos; mas, apesar disso, o sexo desprotegido foi relatado como o principal motivo de utilização da contracepção de 
emergência, seguido do esquecimento de tomada do anticoncepcional de rotina, gerando preocupação em relação à proteção contra infecções sexualmente transmissíveis, bem como uso repetitivo do contraceptivo de emergência e ocorrência de gravidez não planejada.

Nesse contexto, pode-se afirmar que os serviços de atendimento farmacêutico disponibilizados ao consumidor/cliente e farmácias, como estabelecimentos de saúde, inseridos no contexto da atenção primária, podem viabilizar o fornecimento de subsídios para dados epidemiológicos e auxiliar no processo do cuidado das pessoas, bem como na utilização adequada dos medicamentos.

\section{AGRADECIMENTOS}

Nossos agradecimentos ao Serviço de Atendimento Farmacêutico (SACFARMA) de uma grande rede de farmácias comunitárias, e à farmácia filial, dessa mesma rede, pela disponibilização para a execução do estudo. Também, agradecemos a Francisco José Batista de Lima Júnior, pela valiosa contribuição na elaboração dos gráficos desse trabalho.

\section{REFERÊNCIAS}

1. MITTAL S. Emergency contraception - Potential for women's health. The Indian Journal of Medical Research. 2014;140 (Suppl 1):S45-S52. Disponível em: <http://www.ncbi.nlm.nih.gov/pmc/articles/PMC4345752/>. Acesso em: 15/02/2015.

2. CARVAJAL A, et al. Emergency contraceptive pill safety profile. Comparison of the results of a follow-up study to those coming from spontaneous reporting. Pharmacoepidemiol Drug Saf. 2015 Jan; 24(1):93-7. Disponível em: <http://www. ncbi.nlm.nih.gov/pubmed/25408302> Acesso em: 01/01/2016.

3. CONSELHO FEDERAL DE MEDICINA. Resolução CFM nº. 1.811/2006. Estabelece normas éticas para a utilização, pelos médicos, da Anticoncepção de Emergência, devido à mesma não ferir os dispositivos legais vigentes no país. Diário Oficial da União, Poder Executivo, Brasília, DF, 17 jan. 2007. Seção I, p. 72.

4. DELBANCO, S. F. et al. Are we making progress with emergency contraception: recent findings on american adults and health professionals. J. Am. Med. Wom. Assoc., [s.I.], v. 53, p. 242-246, 1998. Disponível em: <http://www.ncbi.nlm.nih. gov/pubmed/9859632>. Acesso em: 23/06/2008.

5. NOGUEIRA AA; REIS FJC \& POLI NETO OB. Anticoncepcionais de emergência - por que não usar? Medicina, Ribeirão Preto, 33:60-63 jan./mar. 2000. Disponível em: <http://www.revistas.usp.br/rmrp/article/view/7631/9157>. Acesso em: $5 / 02 / 2015$.

6. WEBB A \& TABERNER D. Clotting factors after emergency contraception. Adv Contracept 9: 75-82, 1993. Disponível em: $<$ http://www.ncbi.nlm.nih.gov/pubmed/8342458>. Acesso em: 28/10/2009

7. BRASIL. MINISTÉRIO DA SAÚDE. Anticoncepção de Emergência. Manual de Perguntas e Respostas para Profissionais de Saúde, Brasília-DF: Ministério da Saúde, 2011. Disponível em: <http://bvsms.saude.gov.br/bvs/publicacoes/anticoncepcao_ emergencia_perguntas_respostas_2ed.pdf>. Acesso em: 21/02/2012.

8. FIGUEIREDO, R. et al. Comportamento sexual, uso de preservativos e contracepção de emergência por adolescentes do município de São Paulo: estudo com estudantes de escolas públicas de ensino médio. São Paulo, Instituto de Saúde, 2008. 38p. Disponível em: <http://redece.org/compsexual.pdf>. Acesso em: 04/05/2009. 
9. BRASIL. MINISTÉRIO DA SAÚDE. Manual Operacional para Comitês de Ética em Pesquisa. Conselho Nacional de Saúde, Comissão Nacional de Ética em Pesquisa. - 4. ed. rev. atual. Brasília-DF: Editora do Ministério da Saúde, 2008. Disponível em: <http://conselho.saude.gov.br/web_comissoes/conep/aquivos/materialeducativo/Manual_ceps_v2.pdf>. Acesso em: $14 / 08 / 2009$

10. SOUZA, R.A. Pílula do Dia Seguinte: uma revisão de literatura sobre a Anticoncepção de Emergência. Artigo de Revisão. Cadernos UniFOA. Edição no 8, 2008. Disponível em: <http://www.scielo.br/scielo.php?script=sci_ arttext\&pid=S0103-73312012000100002 $>$. Acesso em: 23/10/2009

11. HARDY, E et al. Anticoncepção de emergência no Brasil: facilitadores e barreiras. Cadernos de Saúde Pública (FIOCRUZ), Brasil, v. 17, n. 4, p. 1031-1035, 2001. Disponível em: <http://www.scielo.br/scielo.php?pid=S0102311X2001000400038\&script=sci_arttext>. Acesso em: 23/07/2013.

12. FIGUEIREDO R. Contracepção de Emergência no Brasil: necessidade, acesso e política nacional. Revista de Saúde Sexual e Reprodutiva 2004. N. 13. setembro. Disponível em: <http://www.ipas.org.br/arquivos/10anos/Regina_CE2004.doc.>. Acesso em: 7/010/2015.

13. ARCANJO, C. M.; OLIVEIRA, M.I.V.; BEZERRA, M.G.A. Gravidez em adolescentes de uma unidade municipal de saúde em Fortaleza-Ceará. R Enferm 2007. 11 (3): 445 - 51. Disponível em: <http://www.scielo.br/pdf/ean/v11n3/v11n3a08>. Acesso em: 02/03/2008.

14. FAUS, M.J., MARTINEZ, F. La atención farmacêutica em farmacia comunitaria: evolución de concepos, necesidades de formación, modalidades y estratégias para supuesta en marcha. Pharm. Care Esp. v. 1, p. 56-61, 1999. Disponível em: <http://www.ceatenf.ufc.br/Artigos/ATENFAR\%20e\%20URM\%20Adriano\%20Max.pdf>. Acesso em 29/05/2015

15. Da SILVA, M.R.B. et al. Por que elas não usam?: um estudo sobre a não adesão das adolescentes aos métodos contraceptivos e suas repercussões. Editora Rede Unida v.1, n.4 DOI: http://dx.doi.org/10.18310\%2F2446-4813.2015v1n4p75-83, 2015. Disponível em: <http://revista.redeunida.org.br/ojs/index.php/rede-unida/article/view/633>. Acesso em: 20/05/2015.

16. Comissão Nacional de Incorporação de Tecnologias no SUS, CONITEC. Protocolo Clínico e Diretrizes Terapêuticas Infecções Sexualmente Transmissiveis. Ministério da Saúde, Brasília, DF. Abril, 2015. Disponível em: <http://conitec.gov.br/images/ Consultas/Relatorios/2015/Relatorio_PCDT_IST_CP.pdf>. Acesso em: 10/01/2016. 